\title{
The Use of Economic Models in Managed Care Pharmacy Decisions
}

\author{
Kent H. Summers, Timothy R. Hylan, and Eric T. Edgell
}

\begin{abstract}
In light of concerns about rising pharmaceutical expenditures, managed care organizations call upon clinical pharmacists to include economic factors in their decisions regarding product selections for formulary inclusion. Good decisions increase the likelihood of achieving the desired balance between providing high-quality care and containing overall health care costs. Given time limitations and the wide variety and quantity of data sources available for potential incorporation into decisions, pharmacists often face the risk of making suboptimal decision recommendations to Pharmacy and Therapeutic committees. Pharmacists can use a structured decision-making process that utilizes decision-analytic and health-economic models to optimize these recommendations. Such an approach also permits a strong
\end{abstract}

defense when decisions are questioned. This article provides a framework for understanding, evaluating, and using decision-analytic and predictive-economic models to facilitate good decisions and therapeutic outcomes for patients, in addition to improving the managerial effectiveness of managed care pharmacists.

KEYWORDS: P\&T committees, Formularies, Pharmaceutical product selection decisions, Decision making, Decision-analytic models, Markov models, Econometric models, Outcomes research, Pharmacoeconomic research

J Managed Care Pharm 1998; 4: 42-50

\section{Authors}

KENT H. SUMMERS, R.PH., PH.D., TIMOTHY R. HYLAN, PH.D., and ERIC T EDGELL, PHARM.D., M.S. work for Global Health Economics Research at Eli Lily and Company, Indianapolis, IN.

AUTHOR CORRESPONDENCE: Kent H. Summers, R.Ph., Ph.D., Global Health Economics Research, Eli Lilly and Company, Lilly Corporate Center, mail drop 2646, Indianapolis, IN 46285.

ACKNOWLEDGMENT: The authors would like to thank Bob Browne, M.D., Tom Croghan, M.D., Eric Klein, Pharm.D., and Michael Minshall, M.P.H., for helpful comments.

Copyright $\odot 1998$, Academy of Managed Care Pharmacy, Inc. All rights reserved.
E

mployers and insurers, in response to rising drug costs, have taken an increased interest in curtailing the growth of drug expenditures. Managed care organizations (MCOs) provide a means to control such expenditures through the use of restrictive drug formularies.

Individuals and groups-both inside and outside MCOsclosely scrutinize formulary decisions because of their effects on large patient populations. These decisions are especially difficult within MCOs because many of the pharmaceutical products under consideration are used to treat chronic conditions in which clinical and economic outcomes of therapy are not immediately apparent. Pharmacists are particularly vulnerable to criticism if their recommendations to Pharmacy and Therapeutic (P\&T) committees do not lead to expected outcomes. This is especially problematic when such expectations have not been established at the time of the formulary decision. Further, price changes or the emergence of new information or products can provide ammunition to those who might question previously accepted desions. Given limitations of time and personnel, P\&T committee members may default to an inconsistent approach to making decisions. Suboptimal outcomes are more likely when decisions are variably influenced by political dynamics within the P\&T committee, inappropriate generalizations from clinical studies, and inconsistent weighting of the many information sources.

Economic models are tools MCO pharmacists can use within the context of a structured decision-making process to provide recommendations for formulary decisions. These models can help focus $P \& T$ committee discussions, structure the decision-making process, and establish reasonable expectations of outcomes. Economic models also provide pharmacists a means to manage questions about or criticisms of their decision recommendations. This article provides a framework for understanding, evaluating, and using decision-analytic and predictive-economic models in the decision-making process. 
Table 1. Evolution of Drug Products' Economic Evaluation

\begin{tabular}{|c|c|c|}
\hline Evaluation Metric & Role in Evaluation & Critical Questions \\
\hline$\$$ /unit & First level of pharmacy budget consideration & $\begin{array}{l}\text { - Effectiveness equality? } \\
\text { - Compliance? }\end{array}$ \\
\hline$\$($ or $\mathrm{mg}$ )/day (or month) & $\begin{array}{l}\text { Considers impact of both unit costs and } \\
\text { utilization on the pharmacy budget }\end{array}$ & $\begin{array}{l}\text { - Cross-sectional averages? } \\
\text { - Adjustments for patients severity? } \\
\text { - Prescribing by specialists? } \\
\text { - Relevance of time period? }\end{array}$ \\
\hline \$/episode of care & $\begin{array}{l}\text { May incorporate total direct medical costs } \\
\text { in a clinically relevant period }\end{array}$ & $\begin{array}{l}\text { - Drug utilization patterns? (e.g., concomitant } \\
\text { therapy, switching, premature discontinuations) } \\
\text { - Time periods relevant for chronic diseases? }\end{array}$ \\
\hline$\$ /$ clinical and/or societal outcomes & $\begin{array}{l}\text { Considers all relevant costs and consequences } \\
\text { of pharmaceutical therapy from a broad } \\
\text { perspective }\end{array}$ & $\begin{array}{l}\text { - Difficulty of comprehensive studies? } \\
\text { - Relevance to payors' perspectives? }\end{array}$ \\
\hline
\end{tabular}

\section{MAKING BETTER DECISIONS}

Pharmacists often face a daunting task when trying to secure rational, evidence-based decisions from their institutions' P\&T committees. The political dynamics of P\&T committee members and the difficulty of incorporating multiple sources of product information into a single decision combine to create a tremendous challenge to pharmacists. The consistent use of a structured decision-making process increases the likelihood of making the best formulary decisions.

A major challenge $\mathrm{MCO}$ pharmacists experience when participating in formulary decisions is the difficulty of incorporating economic considerations into decisions traditionally based on clinical data alone. Experience suggests that the first step in this process is the consideration of drug price per unit (see Table 1). However, this aspect considered alone or predominantly in a decision is subject to criticism because of the potentially unwarranted assumption of effectiveness equality when evaluating therapeutic classes. Further, it may not take into account the effect of compliance on drug costs.

Another approach may compare dollars or milligrams consumed per day (or month) by people using the products under consideration. When considering average milligrams per day, we do not know where patients are in a course of therapy because these "snapshot" or cross-sectional figures contain an unknown combination of patients at various points in therapy. Further, this approach may not consider differences in patients' severity of illness. We might expect higher dosages of products to be used to treat sicker patients. Differential usage by prescribing specialists would be one clue we might consider when assessing such evaluation metrics. Finally, the time used in the denominator of a milligram-per-day metric may not be clinically relevant. Decisions driven by this metric, therefore, may not select products providing optimal quality or efficiency of therapy.

Considerations of direct medical costs consumed per episode of care can be viewed as the next level of evaluation. This approach may incorporate differences among products' utilization patterns during the disease episode. The key element of this approach is the selection of a time period that is clinically appropriate. The selection of clinically relevant time periods is easier for acute diseases, such as uncomplicated infections. The selection of episode length becomes much more difficult when considering pharmacotherapy for chronic diseases.

An evaluation metric using dollars per total clinical and societal outcomes is the most comprehensive means of comparing pharmaceutical products. This approach takes into account all relevant costs and consequences of pharmaceutical therapy from a broad perspective (e.g., pharmacoeconomic research). However, such evaluations are difficult to perform due to cost and time considerations. Further, MCO decision makers may question the relevance of considering patient benefits from therapy that may occur beyond their enrollment period (e.g., current cost increases for chronic therapy that produce benefits after patient retirement).

Outcomes studies can either be prospective or retrospective. Prospective economic studies in the naturalistic setting can help decision makers evaluate alternative institutional policy questions. A good example of such a study is Simon et al., ${ }^{1}$ which focused on the question: What are the cost outcomes associated with placing newly diagnosed depressed patients on a tricyclic antidepressant versus a selective serotonin reuptake inhibitor? However, prospective trials are administratively complex, expensive, lengthy, and uncommon in the literature. Retrospective studies rely on data (often from paid claims) collected in natural settings. Although less obtrusive to patients and less administratively complex, the data collected for these studies are not as detailed or precise as those collected in a prospective trial setting. Retrospective outcomes studies often use statistical models to control for confounding effects, selection bias, and other observed and unobserved differences between patients. In the absence of large prospective trials, retrospective analyses can provide decision makers with useful information to improve decisions.

The use of pharmacoeconomic information in product selection offers the possibility of making better decisions 


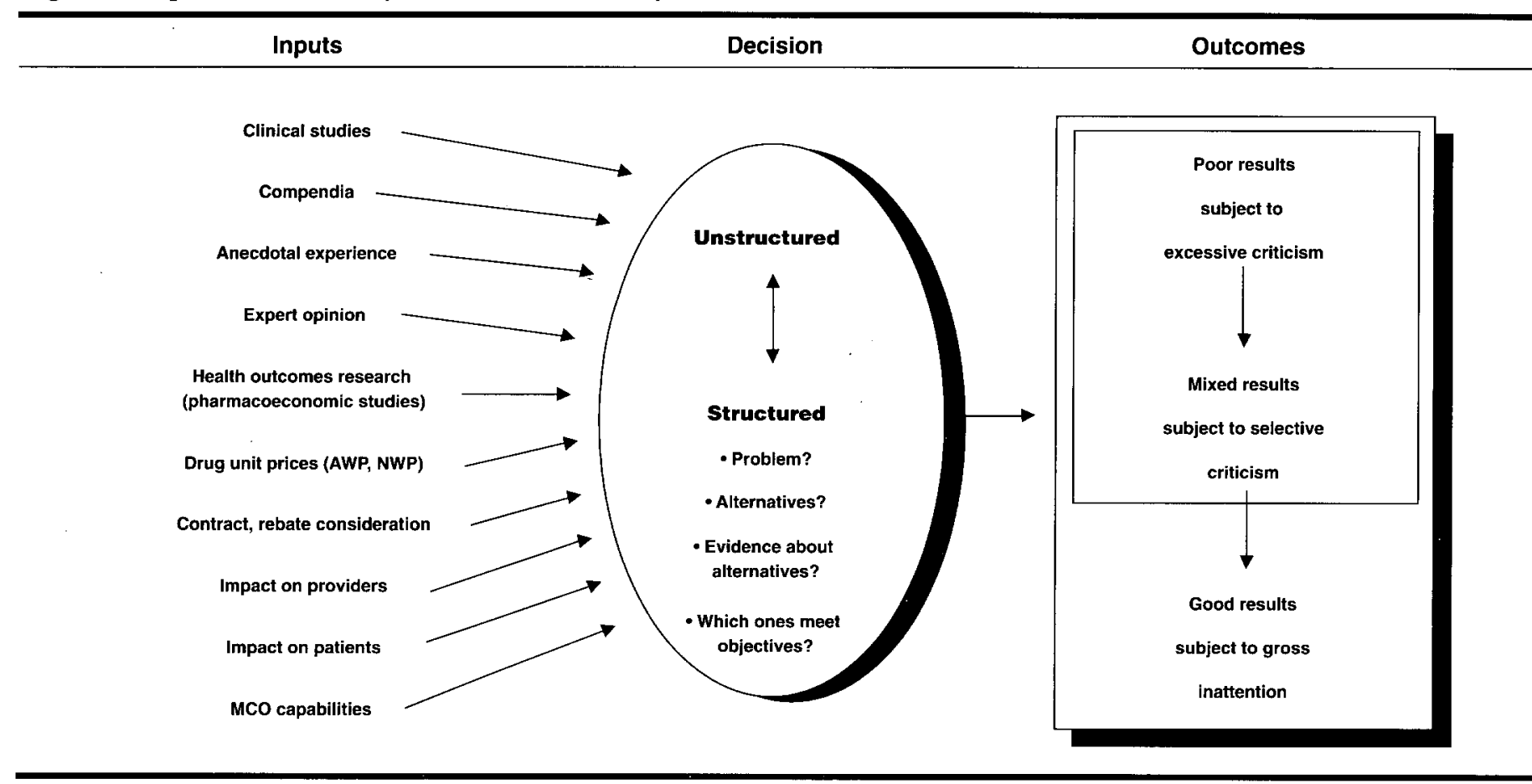

through a more comprehensive (clinical and economic) evaluation of alternatives. However, outcomes studies represent yet another source of information to consider when making product selection decisions. The challenge lies in their rational incorporation into a consistently applied decision-making process. An unstructured approach to decisions increases the likelihood of achieving unfavorable results and provides little defense if they occur (see Figure 1). On the other hand, a structured decisionmaking process utilizes a framework that permits the incorporation of evidence into logical and reproducible decisions on a consistent basis. Thus, a structured approach can yield significant benefits for patients, decision makers, and their MCOs.

\section{UNSTRUCTURED DECISION-MAKING PROCESS}

With the large amount of information and the very limited time and resources available to identify, evaluate, and analyze it adequately, a natural response of many P\&T committee members is to retreat to a simpler, more direct approach to decision making. This is propagated in many cases by limited, conflicting, and/or ambiguous information. When faced with a wide array of information inputs, decision makers may make the often unfounded assumption of equal effectiveness among products in therapeutic classes. Indeed, reviews of the pharmacoeconomic literature have demonstrated that a frequent flaw in studies is the implicit assumption of therapeutic equality among products when ostensibly comparing their cost-effectiveness. ${ }^{2.3}$ This assumption of therapeutic equivalence must be supported by scientific evidence if used as the basis for decision making (i.e., cost-minimization rather than cost-effectiveness analysis).
Further, unstructured decisions often employ arbitrary weights given to the many sources of information input. For example, drug class evaluations may be unduly swayed by recent clinical studies that particularly impress influential P\&T committee members or that support previously developed opinions (i.e., tradeoff contrast fallacy). ${ }^{4}$ Such decisions may not necessarily be based on all of the scientific evidence available. These factors combine to hinder the effectiveness of pharmacists making recommendations to their P\&T committees.

\section{STRUCTURED DECISION-MAKING PROCESS}

A structured, data-driven decision-making process requires addressing the following four key questions:

1. What is the problem we are trying to solve? It is important to state clearly the problem or objective facing the decision makers. P\&T committee members need to decide if their primary focus regarding formulary selection is to:

$\Delta$ minimize the pharmacy budget;

$\Delta$ minimize the total cost of providing care to a

defined population;

$\Delta$ minimize patient exposure to administrative restrictions (as is often the case with employers' self-insured programs or administrative services-only products);

$\Delta$ maximize the effectiveness of the product's use; or

$\Delta$ maximize the quality of care.

In most cases, the problem is multidimensional-MCOs want to achieve several different objectives simultaneously. This is often difficult to accomplish. Decision makers must clearly 
understand and explicitly communicate the trade-offs between competing objectives. If the primary problem is the pharmacy budget, drug unit costs are but one component of consideration. The total pharmacy budget includes drug unit costs multiplied by their utilization. Therefore, drug utilization represents a very important consideration in pharmacy budget problems and may be a crucial issue in controlling costs.

2. What are the alternatives? In other words, what are the available pharmaceutical and nondrug options in therapy? These alternatives might include all possible pharmaceutical products currently available in a therapeutic class. To facilitate formulary decision adoption, the alternatives may include only products achieving some minimum of physician and patient acceptance as indicated by market share. Alternatively, scientific evidence may, in some cases, support the delayed access to medication therapy until diet and exercise failure is documented.

3. What does the evidence say about the available alternatives? Answering this question requires gathering information on the costs and outcomes associated with the therapeutic options. This might include a review of the existing clinical and outcomes research studies, discussions with company representatives, and meetings with opinion leaders (i.e., expert opinion) to understand what the scientific literature says about each of the alternatives under consideration. The thoroughness of evidence identification and evaluation is directly related to the quality of the decision. Economic models represent an important source of scientific evidence to consider in a structured decision-making process. Systematic incorporation of this evidence into product evaluations leads to improved decisions.

4. Which alternatives appear to best address the stated objectives? After identifying and considering all the available evidence, key themes or recurrent findings are likely to emerge that are consistent across a number of studies and are likely to address decision makers' objectives. Products associated with a preponderance of supporting evidence may be most likely to address the stated objectives.

\section{MODELS IN DECISION MAKING}

A structured, thorough decision-making process includes a comprehensive review of the scientific evidence and may include the use of studies based on economic models. Just as decisions are improved through the consistent use of a structured process, economic models become better tools to support this process when used within a systematic framework (see Figure 2). This may involve accepting published healtheconomic models as reasonable surrogates of the local MCO environment. Alternatively, it may involve consolidating information inputs from various sources (e.g., clinical studies, outcomes research, expert opinion) into a synthesized decisionmaking model to predict the economic impact of various decision possibilities. In addition, MCO pharmacists may elect to

Figure 2. Framework for Incorporating Models into Formulary Decisions

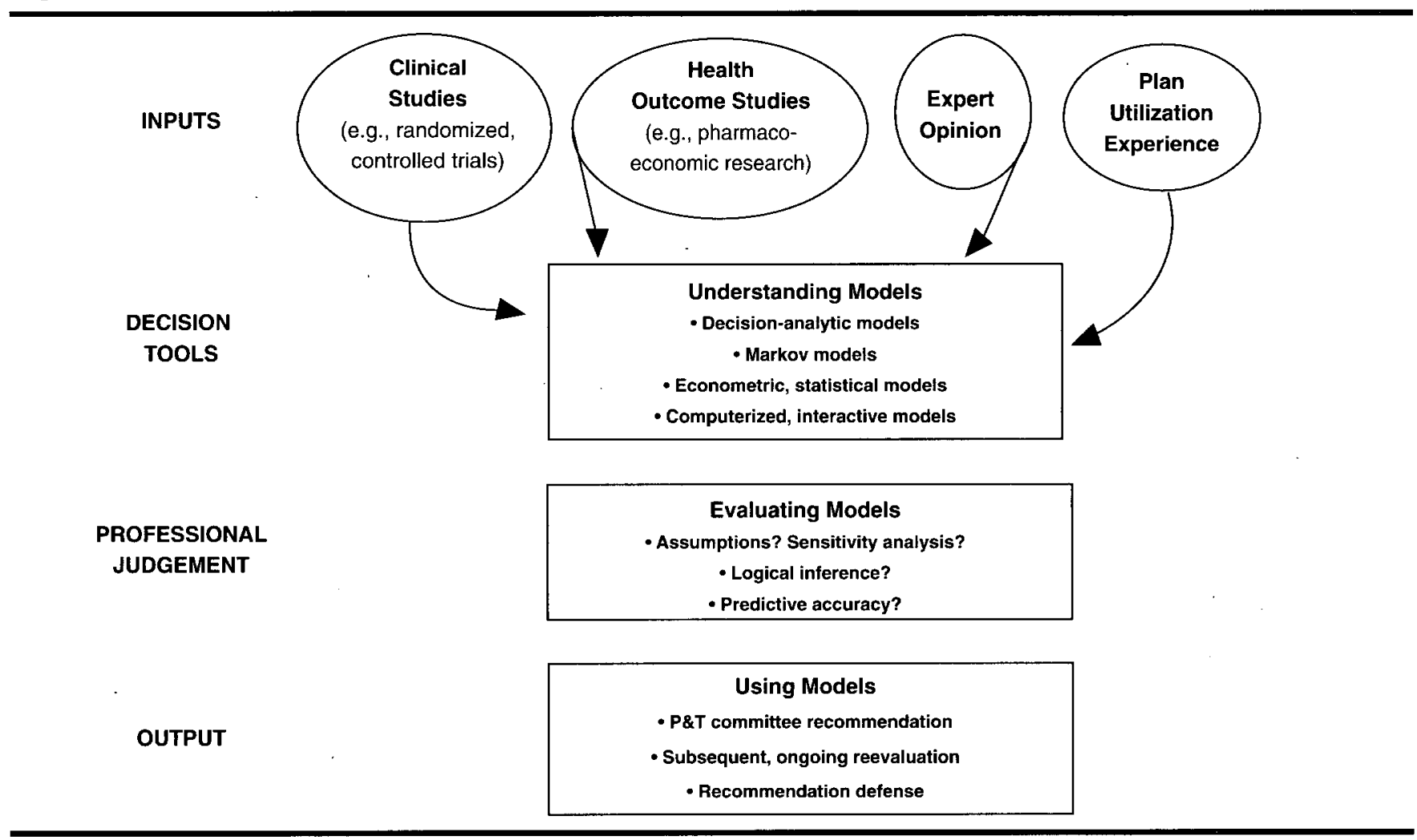


use an interactive computer model that incorporates planspecific utilization data in addition to or in place of data from the published literature. In all cases, professional judgment is needed to select the most appropriate information inputs, choose the best model, and evaluate the accuracy of its predictions.

\section{Understanding Models}

At a very general level, a model is a representation of reality. A child's scale version of an aircraft carrier is a model, as is a flowchart illustrating how the post office moves a letter from one location to another. In other cases, models are used to understand objects or processes we cannot observe. An architect's rendition of a house before it is built is a model, as is a weatherforecasting computer program used to predict tomorrow's weather. Models are also useful whenever observing the actual object or process is difficult, expensive, impractical, unethical, or impossible. They are helpful in simplifying reality to better understand and deal with practical problems.

Models lie at the core of research activities in the social and pharmaceutical sciences. For example, social and behavioral scientists in pharmacy schools use the Health Belief Model to better understand patients' medication compliance behavior ${ }^{5,6}$ In other areas of pharmaceutical science, disease models are used to depict the natural history of disease and to help researchers identify new intervention opportunities. Basic molecular structure models generate testable hypotheses that researchers use to guide the discovery process and to identify new chemical entities (NCEs). In addition, laboratory rats serve as biological models to assess the pharmacokinetic and pharmacodynamic properties of NCEs, when observing the actual process in humans at that very early stage is unethical. Hence, models are used during clinical research and development of products to permit predictions of outcomes when observation of the actual process is not feasible.

\section{Decision-Analytic Models}

Decision-analytic (DA) models are often used in the economic evaluation of pharmaceuticals. These models have used resource utilization data collected during clinical trials, results appearing in the published literature, meta-analyses of clinical studies, and marketplace evidence of similar products to predict new product performance. ${ }^{7-21} \mathrm{DA}$ models quantify the likely value of the pharmaceutical product by analyzing both the benefits and the costs of continuing with a drug's development. Because of the difficulty of collecting actual product performance data in the developmental phase (i.e., Phase III clinical studies under random clinical trial (RCT) protocols), economic models help manufacturers allocate funds by estimating the likelihood of product approval, the size of the market for the compound, likely competitors, and the potential revenue stream generated relative to the costs of research, development, and production. In this way, scarce research and development budgets can be applied to those products that have the greatest potential return on research investment.
DA models can also serve as tools to facilitate product selec-tion decisions. They provide important information on new products when P\&T committees need information to make decisions yet actual experience with the new drug is not available. Thus, DA models are useful when data from actual clinical practice settings do not exist (such as at launch), providing a bridge between available clinical data (demonstrating efficacy in a controlled environment) and the needed effectiveness data.

DA is a modeling technique that identifies altemative pathways and describes the costs and consequences of each. It has been used to develop policies regarding the management of patient populations by providing information regarding which of two or more strategies for approaching a medical problem has the best outcome or the most value. ${ }^{22.23}$ The first step in any DA is to systematically break down a problem into its components and create a decision tree that represents these components and the decision options. Each decision option is associated with an explicitly defined outcome. The probability of the occurrence of these outcomes is then identified through medical literature reviews and/or expert opinions. The value (e.g., quality-adjusted life-year, or QALY) of each outcome is also measured or inferred. Lastly, the decision tree is analyzed statistically to estimate the net value of each decision option.

For example, a P\&T committee may deliberate whether it makes sense to use one of the newer antidepressants as maintenance therapy in depressed patients or to treat acute episodes with an older medication. Such a decision would benefit from considering the DA model by Hatziandreu et al. ${ }^{24}$ They modeled the likely lifetime consequences of two hypothetical cohorts of 35-year-old women at high risk for recurrent depression. Model construction and data elements were derived from the literature and two physician panels. The resultant decision tree (see Figure 3) predicted that it would cost \$3,692 in 1991 dollars per additional QALY for sertraline maintenance therapy compared with episodic treatment with doxepin, a tricyclic antidepressant developed in Europe for treatment of depression. This decision-analytic model used a Markov state-transition approach to arrive at its predictions.

\section{Markov Modeling}

A DA framework works well for clinical situations characterized by chance events that occur linearly over a short time. However, when the disease involves events that occur repeatedly or over a prolonged period, the decision tree becomes "bushy," which makes analysis difficult. ${ }^{25}$ Markov modeling provides a more convenient way to represent clinical situations with ongoing risk. It can replace a decision tree outright or can be grafted onto standard decision trees.

There are four steps in a DA that uses a Markov model to represent a process that links a treatment intervention with an outcome. First, the health states to be modeled must be defined. Then, transitions into and out of the states are described. Next, the time horizon of the analysis must be divided into 
Figure 3. Decision Tree (simplified) for Alternative Antidepressant Treatment Strategies

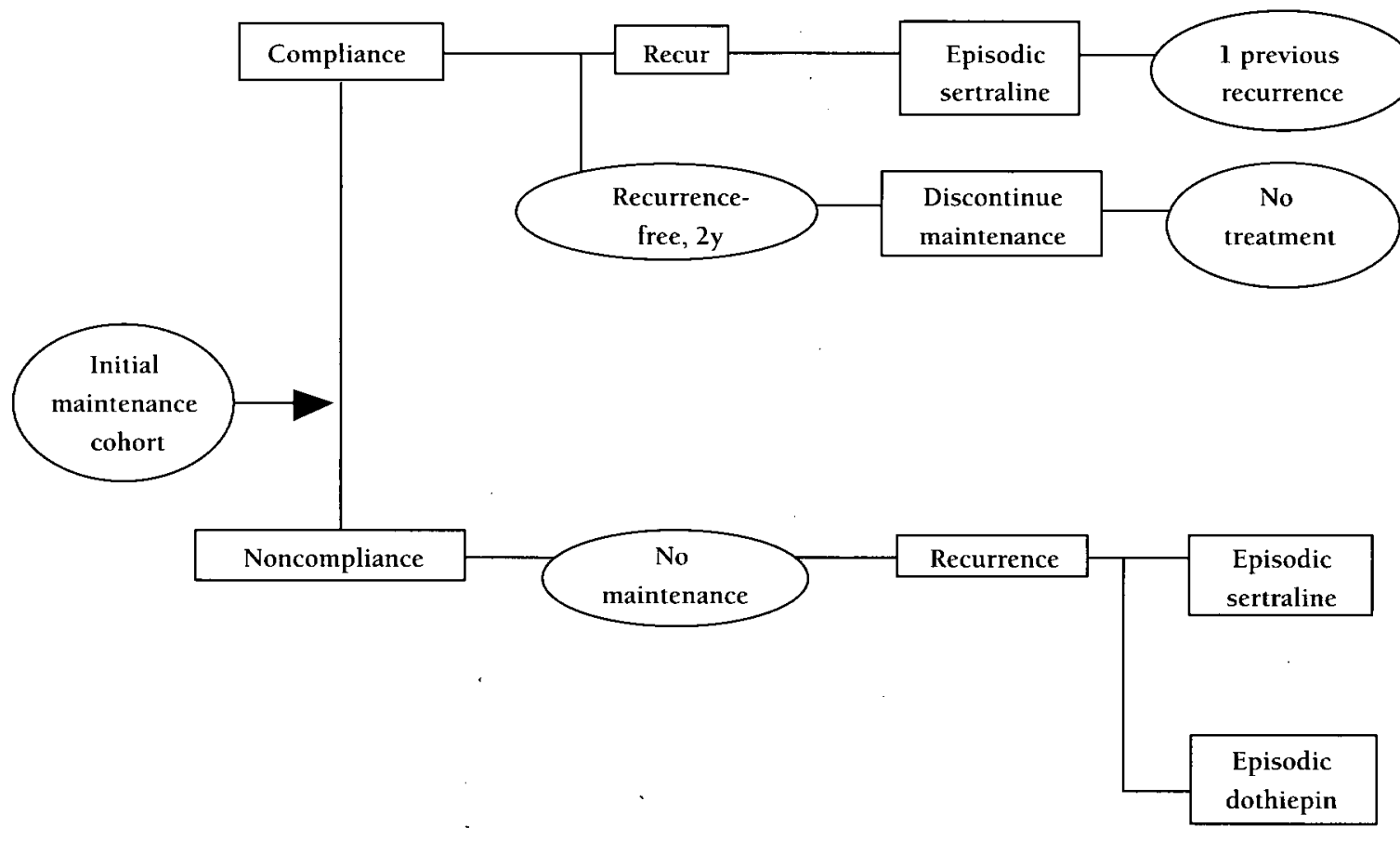

Source: Adapted from Hatziandreu, et al. ${ }^{24}$ (Squares represent events, circles represent states)

equal increments of time, referred to as Markov cycles. During each cycle, the patient may make a transition from one state to another, and it is assumed that a patient in a given state can make only a single-state transition during a cycle. The length of the cycle is chosen to represent a clinically meaningful time interval. Last, the transition probabilities are estimated in the same manner used to estimate other probabilities in a DA.

Figure 4 shows a commonly used representation of the Markov process, called a state-transition diagram, as applied to major depression ${ }^{26}$ The arrows connecting health states indicate the transitions allowed per cycle. Patients starting in a normal state (without symptoms) may remain normal or subsequently require ambulatory treatment. Patients receiving ambulatory treatment for their depression may return to normal, may require continued ambulatory treatment, or may need inpatient care. Evaluation of a Markov model yields the average number of cycles spent in each state. These states may be associated with clinical, economic, or quality-of-life parameters to conduct different types of analyses.

The primary drawback in using Markov models in medical decision making is the "Markovian assumption," which specifies that the behavior of the process subsequent to any cycle depends only on its description in that cycle. ${ }^{27}$ In other words, all patients in a given state at a given time have the same prognosis, regardless of what events led them to that state. Few biological systems fit the Markovian assumption. ${ }^{27}$ In order to overcome this assumption, one must create a separate state for each subset of the cohort that has a distinct utility, cost, and/or prognosis. The Markovian assumption is not strictly followed in medical decision making. However, the assumption is necessary to model predictions with a finite number of Markov states.

\section{Econometric and Statistical Models}

Data used in many outcomes studies are generated from retrospective analyses of administrative claims or other databases that capture patient resource utilization and other outcomes measures. Unlike data from clinical trials, observational data indicate the impact of individuals' (e.g., patients, physicians) decisions in actual clinical practice. However, these data may be subject to a number of biases including those resulting from nonrandomization, unobserved confounders (selection bias), omitted variables, and measurement error in the observed variables (however, measurement error may also be present in a clinical trial).

Multivariate statistical techniques are used to deal with potential biases encountered in observational data. In econometric analysis, for examplè, multivariate regression techniques are used to estimate the effect of one variable on another, holding other observed and unobserved factors (cofounders) constant. Despite claims to the contrary ${ }^{28}$ many well-developed, sophisticated, and accepted techniques in the econometrics literature can be drawn upon to mitigate invariable problems, selection, and other unobserved biases, as. well as specification problems that arise when working with nonexperimental data. ${ }^{29}$. ${ }^{32}$ There is much experience in the use of econometric methodologies in retrospective database analyses. ${ }^{33}$ However, their application to health care technology evaluation is relatively recent. ${ }^{34}$ The time, cost, and resource advantages of this 
Figure 4. Transition-state Diagram Used in a Markov Process Model

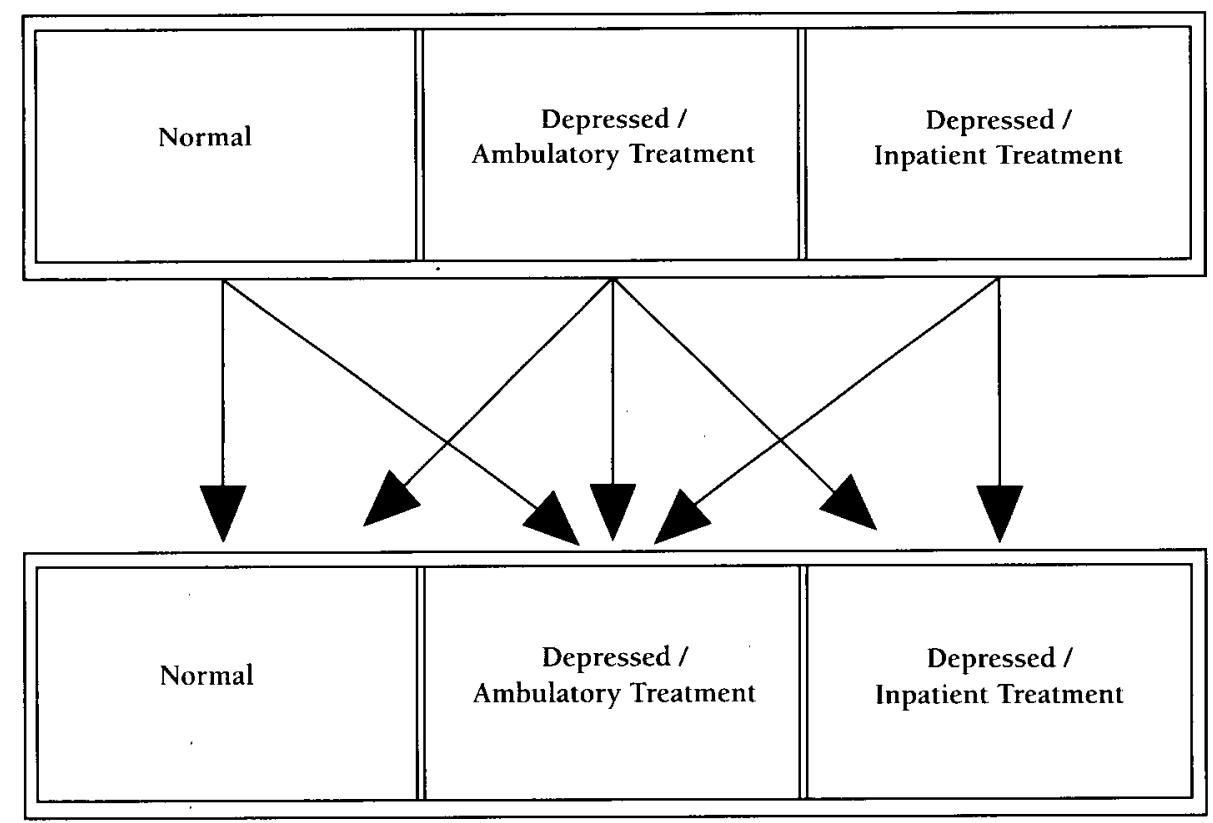

Source: Nuijten MJC, Hardens M, Souetre E. ${ }^{26}$

approach relative to a large randomized prospective trial provide attractive opportunities to evaluate the economic impact of pharmaceutical products in the market. Examples of MCOs conducting these studies suggest that they may be useful to these organizations. ${ }^{35}$

Two recent retrospective database studie ${ }^{36,37}$ used statistical techniques to control for unobserved factors that might be correlated with both initial antidepressant selection and health care expenditures. In these two papers, a two-stage econometric model was used to estimate the one-year total direct health care expenditures associated with the use of different antidepressants, while controlling for other factors that may also explain the results. In the first stage, the probability that a patient received a particular antidepressant was estimated. In the second stage, total direct health care expenditures were estimated, adjusting for other observable factors (e.g., age, gender, comorbid diseases) as well as the estimated probability of antidepressant selection from the first stage. By including this estimated probability, initial product selection factors that may bias the results are controlled. This statistical model mitigates the impact of sample selection bias on the results of retrospective claims database studies. It serves much the same purpose of random patient assignment to treatment groups used in prospective clinical studies.

\section{Interactive Models}

A critical assumption of DA models is that the MCO's population is similar to the study population used in the baseline analysis. If MCO pharmacists do not feel comfortable with this assumption, they can first conduct an analysis using data specific to their own population and project the likely impact of the new drug using those parameter estimates. Computerized spreadsheets permit findings from econometric analyses to be used in actual settings. In these interactive models, MCO pharmacists insert their own input costs and resource utilization data into a series of spreadsheets. Then, the likely resource utilization and total cost implications of using a proposed drug are determined using baseline parameter estimates (sometimes from an econometric model).

These interactive models thus accommodate data from MCOs' databases. For example, in the case of the choice of antidepressant therapy, a decision tree helps to map out a health plan's existing cost structure for the treatment of a disease. Once done, the DA model simulates the cost and other consequences of switching to different products or moving to a new class of intervention (e.g., psychotherapy) within the depression disease state. In addition, either the proportions of patients utilizing a particular therapy, the intensity or frequency of the therapy, or the unit costs are varied to reflect differences that may occur.

Thus, economic models can be used to accommodate the various environments faced by MCO pharmacists. This flexibility produces three important benefits. First, because of the differences between RCTs and clinical practice, tests of economic models in clinical practice can provide additional evidence regarding a drug's anticipated value in an actual environment. Second, interactive models permit pharmacists to use their own data in projecting the likely effect of a drug in their particular situation. Finally, economic models can be continually retested in actual settings to provide additional evidence of 
their predictive ability, providing confidence in decisions based on the model.

\section{Evaluating Models}

The use of economic models to demonstrate drug product performance has been questioned. Concerns include the seemingly large set of methodologies used to evaluate costs and outcomes in actual practice, the assumptions of the model, and the relevance of the model to particular settings. ${ }^{38}$ In economic models, the focus is generally on just a few decision variables and factors that influence decisions. Individuals make decisions about many different things simultaneously, and these factors influence decision making. Like all models, the value of economic models is derived from their predictive ability, which is ascertained only through repeated testing of the model's predictions. An economic model cannot and does not have to capture every decision variable or factor that may affect physicians' prescribing and patients' utilization decisions. If the economic model consistently predicts what we observe, then it is valuable and useful.

Generally, models have three components that can serve as the basis for evaluating their value in decision making:

A Input assumptions are conditions deemed reasonably representative of the environment. This evaluation creates the question: Are the inputs into the model reasonably representative of reality? Economic models, like all models, rely upon a set of assumptions in development. An implicit assumption of DA is that the values of the probabilities and of the utility measures are the correct values for these variables. Pharma-cists should question whether the data inputs in the model make sense. The use of expert opinion as a driver of model output, in particular, should be questioned. Expert opinion is often used in cases in which adequate data are not available for input. One should question the weight of this input, particularly when experts are asked to estimate patient preferences. The reasonableness of these and other input assumptions can be tested with sensitivity analyses. In sensitivity analysis, key parameters of the model are varied to determine how much the results change given alternative assumptions. When a conclusion of an economic model is shown to be invariant to a relevant range of assumptions, confidence in the conclusions of the analysis is enhanced. ${ }^{39}$ For example, DA models often make assumptions about treatment effectiveness by relying on dropout rates reported in clinical trials to predict the costs of treatment failure. Dropout rates from clinical trials due to adverse events may differ from dropout due to lack of efficacy, resulting in different costs of treatment failure. ${ }^{40} \mathrm{~A}$ finding of a DA model that is invariant to different assumptions about dropout rates and their impact on the costs of treatment failure would be more robust than a finding that differed depending on the dropout rate assumptions. This is the reason for using sensitivity analysis to evaluate the strength of conclusions drawn from economic models.

A Logical inference is the process that develops the model. This is generally a process of deductive reasoning that uses the model's assumptions to develop the structure of the model. This leads to the question: Is the underlying structure of the model reasonable? Pharmacists should question whether the relationships included in the model and the weightings given to inputs make sense. Clinical experience might suggest that the treatment pathways used in a model are not realistic. Although some variations are likely, the actual provision of care in a particular setting may deviate widely from the underlying structure used in a model. Pharmacists can factor these variations into their acceptance of models used in decisions.

A Predictions are the output estimates generated by the model that are subject to continuing evaluation. This evaluation creates the question: How close did the model's predictions come to actual experience? Models can be informative, but their underlying value lies in their ability to accurately predict reality. ${ }^{41.42}$ These predictions are also referred to as "refutable propositions. ${ }^{143}$ We cannot determine whether a model's predictions are true; it is only possible to determine whether the model's predictions are not refuted. This is a matter of logic because a new model can be developed that can explain the same set of events. In addition, no single test of a model's predictions can determine a model's value. A model's value grows by repeatedly being unable to disprove its predictions. To the extent that a model would have accurately predicted outcomes using retrospective data, MCO pharmacists can have increased confidence that the model will accurately predict future events.

\section{Using Models}

After the available models have been evaluated and selected for use, MCO pharmacists can use them in the P\&T committee's decision-making process. Their use necessitates the explicit consideration and weighting of information inputs into the decision. Thus, models represent a tool to gain P\&T committee members' agreement regarding information inputs and the decision-making process. Models can form the basis to focus discussions during P\&T committee deliberations, saving time through the avoidance of discussions about issues that are neither included in the model nor relevant to product selection. As changes (new products, prices, utilization patterns, or information) appear, interactive models can be recalculated to incorporate these latest inputs. This facilitates making difficult decisions regarding whether to stay with the original product selected or to switch to a new one. Confidence in the decisionmaking process benefits from ongoing reevaluation of the model's predictive ability.

The rationale used in the model serves as the basis for defense when parties internal or external to the MCO question the wisdom of decisions. In such situations, the focus of criticism can be directed toward the decision-making process rather than the pharmacist involved with the decision. Also, pharmacists can use models to gain consensus regarding reasonable expectations of decision outcomes. This permits satisfaction management as internal (P\&T committee members) and external (employers, physicians, patients) MCO stakeholders 
understand what is reasonable to expect from decisions. Thus, the use of economic models permits pharmacists to increase the likelihood of good decisions, as well as to anticipate, avoid, and deflect criticism of decision'recommendations that are questioned.

\section{CONCLUSIONS}

Models are potentially useful tools for MCO pharmacists who must make timely recommendations to P\&T committees regarding formulary decisions. Models can play a key role in a structured, evidence-based decision-making process as a way for P\&T committees to make better, more defensible decisions and improve their competitive edge in today's health care marketplace: An important step in this process is reviewing the available evidence from outcomes studies that often rely upon economic models. When consistently used, this process can improve therapeutic outcomes for patients and managerial effectiveness for MCO pharmacists.

\section{References}

1. Simon $\mathrm{G}$, VonKorf $\mathrm{M}$, Heiligenstein $\mathrm{JH}$, et al. Initial antidepressant choice in primary care: effectiveness and cost effectiveness of fluoxetine vs. tricyclic antidepressants. JAMA 1996; 275(24): 1897-1902.

2. Udvarhelyi S, Colditz GA, Ria A, et al. Cost effectiveness and cost benefit analysis in the medical literature. Ann Int Med 1992; 116(3): 238-44.

3. Lee JT, Sanchez LA. Interpretation of cost-effective and soundness of economic evaluations in the pharmacy literature. Am J Hos Pharm 1991; 48(12): 2622-27. 4. Summers KH, Szeinbach SL. Formularies: the role of Pharmacy and Therapeutics (P\&T) committees. Pharmacy \& Therapeutics 1995; 20(8): 534-46. 5. Rosenstock IM. Why people use health services. Milbank Memorial Fund Quarterly 1966; 44(3-Sup): 94-127.

6. Smith MC, Wertheimer AI, eds. Predicting and detecting noncompliance. In: Social and behavioral aspects of pharmaceutical care, chapter 13, New York: Pharmaceutical Products Press, 1996.

7. Copi I. Introduction to Logic, Fourth Edition. New York: Macmillan, 1972.

8. Anton SF, Revicki DA. The use of decision analysis in the pharmacoeconomic evaluation of an antidepressant: a cost-effectiveness study of nefazodone. Psychopharmacology Bulletin 1995; 31(2): 249-58.

9. Edelson JT, Tosteson AN, Sax P. Cost-effectiveness of misoprostol for prophylaxis against nonsteroidal antiinflammatory drug-induced gastrointestinal tract bleeding. JAMA 1990; 264(1): 41-47.

10. Freedberg KA, Hardy WD, Tosteson ANA, et al. Validating literature-based models with direct clinical trial results: the cost effectiveness of secondary prophylaxis for PCP in AIDS patients. Med Decis Making 1996; 16(1): 29-35. 11. Glick $H$, Kinosian B, Schulman $K$. Decision analytic modeling: some uses in the evaluation of new pharmaceuticals. Drug Information Journal 1994; 28(3): 691-707.

12. Stergachis A. Overview of cost-consequence modeling in outcomes research. Pharmacotherapy 1995; 15(5 pt. 2): 40S-42S.

13. Gabriel SE. Economic evaluation using mathematical models: the case of misoprostol prophylaxis. Journal of Rheumatology 1995; 22(7): 1412-14. 14. Simpson KN. Modeling with clinical trial data: moving from the data researchers have to the data decision makers need. Drug Information Journal 1995; 29(4): 1431-40.

15. Rittenhouse R, O'Brien B. Threats to the validity of pharmacoeconomic analyses based on clinical trial data. In: B. Spilker, ed. Quality of life and pharmacoeconomics in clinical trials, Second Edition. New York, NY: Raven Press, 1996, 1215-23

16. Shiell A, Briggs A, Farrell GC. The cost effectiveness of alpha interferon in the treatment of chronic active hepatitis C. Med J Aust 1994; 160(5): 268-72. 17. Revicki D, Brown RE, Palmer W, et al. Modeling the cost effectiveness of anti-depressant treatment in primary care. PharmacoEconomics $1995 ; 8(6)$ : $524-40$

18. Lowe FC, MCDaniel RL, Chemeil JJ, et al. Economic modeling to assess the costs of treatment with finasteride, terazosin, and transurethral resection of the prostate for men with moderate to severe symptoms of benign prostatic hyperplasia. Urology 1995; 46(4): 477-83.

19. Meltzer HY, Cola PA. The pharmacoeconomics of clozapine: a review. Journal of Clinical Psychiatry 1994; 55(Sup B): 161-65.

20. Minshall M, Dawson A. Integrating a global health economics study within a Phase III clinical trial. Drug Information Journal 1995; 29(4): 1191-99.

21. Adelman JU, Von Seggern R. Cost considerations in headache treatment. Part 1: prophylactic migraine treatment. Headache 1995; 35(8): 479-87. 22. Weinstein MC, Fineberg HV. Clinical decision analysis. Philadelphia, PA: Saunders Company, 1980.

23. Petitui DB. Meta-analysis, decision analysis, and cost-effectiveness analysis: methods for quantitative synthesis in medicine. New York: Oxford University Press, 1994.

24. Hatziandreu EG, Brown RE, Revicki DA, et al. Cost utility of maintenance treatment of recurrent depression with sertraline versus episodic treatment with dothiepin. PharmacoEconomics 1994; 5(3): 249-64

25. Beck JR, Pauker SG. The Markov process in medical prognosis. Med Decis Making 1983; 3(4): 419-58.

26. Nuijten MJC, Hardens M, Souetre E. A Markov process analysis comparing the cost effectiveness of maintenance therapy with citalopram versus standard therapy in major depression. PharmacoEconomics 1995; 8(2): 159-68.

27. Sonnenberg FA, Beck JR. Markov models in medical decision making: a practical guide. Med Decis Making 1993; 13(4): 322-38.

28. Sheldon TA. Problems of using modeling in the economic evaluation of health care. Health Econ 1996; 5(1): 1-11.

29. Greene W. Econometric analysis, Second Edition. New York: MacMillan, 1993

30. Maddala GS. Econometrics. New York: MacMillan, 1988.

31. Heckman JJ. Sample selection bias as a specification error. Econometrica 1979; 47(1): 153-61.

32. McClellan M, McNeil BJ, Newhouse JP. Does more intensive treatment of acute myocardial infarction in the elderly reduce mortality? JAMA 1994;

272(11): 859-66

33. Phelps C. Health Economics. New York: HarperCollins, 1992.

34. Bootman JL, Townsend RJ, McGhan WF, eds. Principles of pharmacoeconomics. Cincinnati, OH: Harvey Whitney Books, 1991.

35. Katzelnick DJ, Kobak KA, Jefferson JW, et al. Prescribing patterns of antidepressant medications for depression in an HMO. Formulary 1996; 31: 374-88. 36. Croghan TW, Lair T]; Engelhart M, et al. Effect of antidepressant therapy on health care utilization and costs in primary care. Psychiatri Serv 1997; 48 (11): 1420-25

37. Hylan TR, Crown WH, Meneades L, et al. SSRI and TCA antidepressant selection and health care costs: a multivariate analysis. J Affect Disord, in press. 38. Wechsler J. The FDA position on cost-effectiveness: a reporter's view. Drug Information Journal 1995; 29(4): 1495-97.

39. Davis D, Holt C. Experimental economics. Princeton, NJ: Princeton University Press, 1993

40. Hotopf M, Lewis G, Normand C. Are SSRIs a cost-effective altemative to tricyclics? Br J Psychiatry 1996; 168(4): 404-09

41. Popper KR. The logic of scientific discovery London: Hutchinson, 1959.

42. Friedman M. The methodology of positive economics. In: Essays in positive economics. Chicago, IL: University of Chicago Press, 1953.

43. Silberberg $E$. The structure of economics: a mathematical analysis. New York: McGraw-Hill, 1978 